\title{
FUNGAL MULTISINUSITIS WITH INTRACRANIAL EXTENSION
}

\author{
Thapa N. ${ }^{1}$ \\ Sinha B. K. ${ }^{2}$ \\ Pradhan B. \\ Bhusal C. L. ${ }^{6}$ \\ Amatya R. C. M. ${ }^{3}$ \\ Shrivastav P. ${ }^{4}$ \\ Baskota K. ${ }^{7}$ \\ Guragain R. P. ${ }^{8}$
}

\section{ABSTRACT:}

Aspergillosis is the commonest fungal infection of nose and paranasal sinuses. Its invasive form is rare in a healthy patient. A case of fungal multisinusitis with intracranial extension with characteristic computerized tomographic finding is reported.

\section{Key Words: Aspergillosis, intracranial extension, CT scan, external frontoethmoidectomy.}

\section{INTRODUCTION:}

Aspergillosis is the commonest fungal infection of nose and paranasal sinuses. ${ }^{4}$ Aspergillus fumigatus being the commonest fungus causing about $90 \%$ of aspergillosis. Aspergillus is a filamentous fungus with septate hyphae in dichotomatous branching at an 45 degree. ${ }^{4}$ Depending upon the invasiveness of the disease aspergillus infection of nose and paranasal sinuses had been classified as follows:-
1. Non-invasive :- This form is characterised by aspergilloma or fungal ball. Nose and paranasal sinuses are full of green-brown sludge. In radiograph the characteristic feature will be heavy metal deposits.

2. Allergic Aspergillus sinusitis :- It is due to immune response to Aspergillus antigens, occuring in young adults with history of asthma or nasal polyps. Nasal discharge contains thick inspissated mucus, eosinophils \& charcot leyden

1. Resident, Department of ORL and Head \& Neck Surgery, TUTH, Maharajgunj, Kathmandu.

2. Asso. Professor, Department of ORL and Head \& Neck Surgery, TUTH, Maharajgunj, Kathmandu.

3. Professor and Head of Dept., Department of ORL and Head \& Neck Surgery, TUTH, Maharajgunj, Kathmandu.

4. Professor, Department of ORL and Head \& Neck Surgery, TUTH, Maharajgunj, Kathmandu.

5. Lecturer, Department of ORL and Head \& Neck Surgery, TUTH, Maharajgunj, Kathmandu.

6. Lecturer, Department of ORL and Head \& Neck Surgery, TUTH, Maharajgunj, Kathmandu.

7. Lecturer, Department of ORL and Head \& Neck Surgery, TUTH, Maharajgunj, Kathmandu.

8. Lecturer, Department of ORL and Head \& Neck Surgery, TUTH, Maharajgunj, Kathmandu.

Address for correspondence : Dr. Narmaya Thapa, Institute of Medicine, Maharajgunj

P.O. Box: 1524, Kathmandu, Nepal.

Tel.: 486008 Ext. 235 (off.), 257205 (Res.)

email: nmc@mos.com.np 
crystals. Fungal hyphae may be identified by spe- have history suggestive of any systemic illness. cial stain (grocott silver stain).

3. Invasive :- This form of aspergillosis is uncommon \& behaves like malignant neoplasm. Proptosis is a prominent feature. It is slowly progressive and locally destructive. Bony destruction can be seen in radiograph. Serological and skin tests will be positive.

4. Fulminant :- This form of aspergillus infection occurs in immuno-compromised patients. It is rapidly progressive and angioinvasive. Intracranial extension is either direct or blood borne. Histological examination will show infiltrating aspergillus hyphae with minimal inflammation.

In all forms the diagnosis can be made from fresh nasal scrapping, mucosa $\&$ biopsies of sinus mucosa.

Non-invasive disease can be managed by surgical debridement \& sinus ventilation. Allergic aspergillus sinusitis may be treated by surgical debridement with systemic steroids. Invasive or fulminant disease requires radical surgery in the form of exenteration of the affected area combined with intravenous amphotericin or oral ketoconazole or intraconazole. ${ }^{4}$

Though fungal sinusitis limited in the sinuses has often been encountered \& treated with Functional Endoscopic Sinus Surgery (FESS) for last few years in our setting, this is the only case of fungal sinusitis with intracranial extension presented so far. Though computerized tomogram showed characteristic heavy metallic (calcium) deposits, fungal stain \& culture came out to be negative.

\section{CASE:}

A 31 year old male presented to ENT. OPD at TUTH with complaint of progressive nasal obstruction in the left side for last 6 months. He did not

On examination, there were multiple polypoidal pearly white masses in (L) middle meatus, insensitive to touch \& no bleeding, septum was deviated to the $(\mathrm{R})$. He also had mild protosis on the left side.

Routine investigations were within normal limit. Plain \& contrast enhanced CT scan of nose \& paranasal sinuses revealed hyperdense nonenhancing mass involving (L) nasal cavity, (L) ethmoid, (L) frontal sinus \& (L) sphenoid. (L) fovea ethmoidalis \& posterior table of (L) frontal sinus was eroded with extension of mass into the frontal lobe with characteristic calcium deposits. (B) maxillary sinuses were hazy but the density was quite different from the mass \& was reported as aspergilloma of paranasal sinuses with intracranial extension (Figure).

\section{Fig. 1}

Keeping in mind of these findings \& diagnosis (L) external frontoethmoidectomy $+(\mathrm{R})$ FESS was done under G/A on 056/8/5. Peroperative findings were :-

- Polyp in (L) middle meatus.

- Extensive fungal debris in (L) ethmoids, frontal \& sphenoid sinuses.

- $\quad(\mathrm{R})$ maxillary sinus was filled with mucopus.

- $\quad$ Roof of (L) ethmoid \& posterior table of (L) frontal sinus was eroded. 
The fungal ball was sent for fungal staining \& culture but revealed no growth. Postoperative period was uneventful.

He had similar problem 5 years back, subsided after surgery in Gandaki zonal hospital 4 years ago where debulking \& debridement of necrotic tissue, bilateral intranasal ethmoidectomy, bilateral antral wash and bilateral middle meatus antrostomy was done under G/A on 052-5-27. Peroperative findings were:-

- Small polyps in bilateral middle meati.

- Very extensive friable necrotic tissue in all $\mathrm{R}+\mathrm{L}$ ethmoid \& sphenoid sinuses.

- Frank pus in (L) antrum.

- Cholesteatoma like cheesy material in ethmoid cells. Histopathological report revealed benign nasal polyp.

\section{DISCUSSION:}

As mentioned above aspergillosis is the commonest fungal infection of nose and paranasal sinuses. It may vary from simple noninvasive form to fulminant type. Computerised tomography will be the investigation of choice for diagnosis, to determine the extent of disease, bony destruction and intracranial extension.

Stammberger et. al. reported that CT findings were almost pathognomonic for culture positive Aspergillus sinusitis in their patients. ${ }^{3}$ However, in our case both fungal $(\mathrm{KOH})$ preparation \& culture came out to be negative.

Though there are various reports of primary frontal sinus aspergilloma presented with proptosis in non-immunocompromised patients, intracranial extension is quite rare in such patients. Our patient, though he was non-immunocompromised, had fungal sinusitis with intracranial extension.

A. Coulthard et. al. have reported a case of frontal lobe aspergilloma as intracranial complication of paranasal aspergillosis. ${ }^{1}$ Similarly, H. Satoh et. al. reported a case of invasive aspergilloma of the base frontal lobe with eventual occlusion of the right internal carotid artery and death.

In our case multipal sinuses (ethmoid, frontal and sphenoid) of the left side along with left nasal cavity were involved with extension of infection in to the frontal lobe through the posterior table of the left frontal sinus.

\section{CONCLUSION:}

Aspergillosis of nose and paranasal sinuses may present either as a simple polyp or may mimic malignancy. Early diagnosis and surgical debridement is necessary. For fulminant or invasive form radical surgery is the must.

\section{ACKNOWLEDGEMENT:}

I would like to express my sincere thanks to $\mathrm{Mr}$. Yogya Raj Rai for his help in typing this article.

\section{REFERENCES:}

1. Coulthard A, Gholkar A, Sengupta RP. Case repot: frontal aspergilloma - a complication of paranasal aspergillosis. Clin Radiol 1991 Dec; 44(6): 425-7.

2. Satoh H, Uozumi T, Kiya K, Ikawa F, Kurisu K, Sumida M, Nakahara A. Invasive aspergilloma of the frontal base causing internal carotid artery occlusion. Surg. Neurol 1995 Nov.; 44(5): 483-8.

3. Stammberger H, Jaske R, Benfort F. Aspergillosis of the paranasal sinuses. Ann Otal Rhinol Laryngol 1984; 3: 251-256.

4. Weir N, Golding-Wood DG. Infective rhinitis and sinusitis In: Scott-Brown's Otolaryngology (Rhinology) $6^{\text {th }}$ edition. AG. Kerr ed. 1997; 41-43. 